\title{
Osteosarcoma of the Breast in a Patient Derived Orthotopic Xenograft (PDOX) Mouse Model Is Arrested by both Cisplatinum and Eribulin
}

\author{
NORIYUKI MASAKI ${ }^{1,2,3,5}$, NATHANIEL F. WU ${ }^{1,4}$, YUSUKE AOKI ${ }^{1,5}$, \\ JUN YAMAMOTO $^{1,5}$, JUN MIYAZAKI ${ }^{3}$ and ROBERT M. HOFFMAN ${ }^{1,5}$ \\ ${ }^{1}$ AntiCancer Inc, San Diego, CA, U.S.A.; \\ ${ }^{2}$ Graduate School of Medicine, International University of Health and Welfare, Tokyo, Japan; \\ ${ }^{3}$ Department of Urology, School of Medicine, International University of Health and Welfare, Narita, Japan; \\ ${ }^{4}$ Department of Molecular and Cellular Biology, Harvard University, Cambridge, MA, U.S.A.; \\ ${ }^{5}$ Department of Surgery, University of California, San Diego, CA, U.S.A.
}

\begin{abstract}
Background/Aim: Primary osteosarcoma of the mammary gland is a very rare disease, accounting for less than $1 \%$ of all mammary malignancies. There is no established firstline treatment and the prognosis is poor compared to normal breast cancer. We previously established the first patient tumorderived animal model of this disease, grown subcutaneously in nude mice. In the present study, we established a patient derived orthotopic xenograft (PDOX) model of osteosarcoma of the breast and investigated the efficacy of cisplatinum $(C D D P)$ and eribulin (ERB). Materials and Methods: PDOX models of primary osteosarcoma of the breast were divided into 3 groups (5-6 mice per group): untreated control; CDDP treatment; ERB treatment. The tumor volume in the 3 groups was compared after 2 weeks. Results: There were significant differences between control and $C D D P$, and control and ERB $(p=0.036,0.046$, respectively). However, there was no significant difference between CDDP and ERB ( $p=0.964)$. Conclusion: $C D D P$ and ERB are candidates for first-line clinical therapy of primary osteosarcoma of the breast.
\end{abstract}

Primary osteosarcoma of the mammary gland is a rare disease that accounts for $0.2-1 \%$ of all breast malignancies (1-3). Because the number of cases is extremely small, no first-line treatment has been determined. The 5-year survival rate for

This article is freely accessible online.

Correspondence to: Robert M. Hoffman, Ph.D., AntiCancer Inc, 7917 Ostrow St, San Diego, CA, 92111, U.S.A. Tel: +1 8586542555, Fax: +1 8582684175, e-mail: all@anticancer.com

Key Words: Breast osteosarcoma, efficacy, cisplatinum, eribulin, first-line, PDOX, patient-derived orthotopic xenograft, nude mouse. this disease has been reported to be $38 \%$ (1), and the prognosis is worse than breast carcinoma which has a 5-year survival rate of $90.2 \%$ (4). Therefore, there is a need to establish the first-line treatment for primary osteosarcoma of the mammary gland.

Cisplatinum (CDDP) is a platinum complex, which was introduced into clinical practice for cancer in the 1970s and is indicated for the treatment of a wide variety of cancers (5). CDDP is still first-line treatment for osteosarcoma of the bone (6). Eribulin (ERB), a halichondrin-based inhibitor of microtubule dynamics, with a novel mechanism of action, targets the microtubule plus-end and destabilizes microtubules by inhibiting microtubule elongation $(7,8)$. In clinical practice, ERB treatment of soft tissue sarcoma has been initiated and has shown some efficacy (9).

In our previous study, we showed that ERB is effective in PDOX mouse models of primary bone osteosarcoma (10). We recently established a nude mouse model of mammary primary osteosarcoma growing subcutaneously (11). In the present study we established a patient-derived orthotopic xenograft (PDOX) mouse model of primary osteosarcoma of the breast to determine the efficiency of CDDP and ERB.

\section{Materials and Methods}

Mice. Athymic (nu/nu) nude mice (AntiCancer, Inc., San Diego, CA, USA), 4-6 weeks old, were used in the present study. All mouse experiments were performed with an AntiCancer Inc. Institutional Animal Care and Use Committee (IACUC)-protocol specifically authorized for the present study. The National Institutes of Health (NIH) guidelines for the Care and Use of Animals under Assurance Number A3873-1 were followed for the use of the mice. The detailed procedures and methods of anesthesia, surgery, and mouse breeding follow previously described methods for the establishment of PDOX (11). 


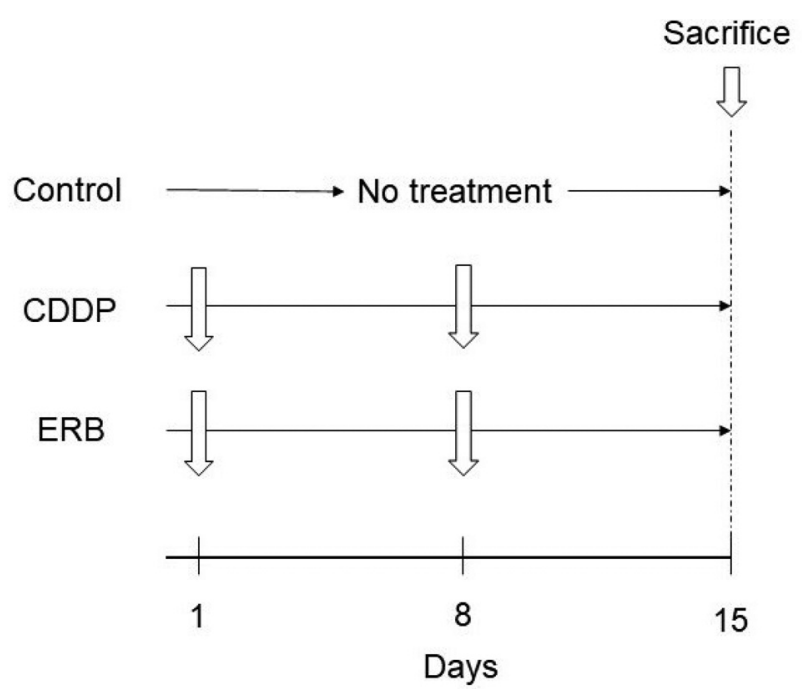

Figure 1. Treatment scheme of the breast-osteosarcoma PDOX nudemouse models. CDDP: cisplatinum; ERB: eribulin.

Patient-derived tumor. The primary osteosarcoma of the breast was previously obtained from the Kaiser Permanente San Diego Medical Center with an approved protocol (IRB\#12617) (11).

Establishment of the PDOX breast osteosarcoma model. A 5-mm incision was made on the skin above the left mammary tissue to create a pocket for the tumor. A 3-4 $\mathrm{mm}$ tumor fragment was inserted into the pocket, and the skin was sutured with a single ligation of a 4-0 nylon suture, as previously described (11).

Treatment scheme. Three weeks after orthotopic implantation, the tumor volume reached $50 \mathrm{~mm}^{3}$, and treatment was started. PDOX mouse models of primary osteosarcoma were randomly assigned to the following three groups: control group; CDDP group (6 $\mathrm{mg} / \mathrm{kg}$, once a week, intraperitoneal injection); ERB group (1.5 $\mathrm{mg} / \mathrm{kg}$, once a week, intraperitoneal injection) (Figure 1). The short and long axis of the tumor was measured using a calipers and the mice were weighed once a week. The volume of the tumor was determined as follows: short axis $\times$ short axis $\times$ long axis $/ 2$. All mice were sacrificed on the 15 th day after treatment. The tumors were then collected for histological evaluation.

Hematoxylin and eosin $(H \& E)$ staining. The $\mathrm{H} \& \mathrm{E}$ staining procedure was performed according to standard protocols.

Statistical analysis. All statistical analyses were performed using EZR (Saitama Medical Center, Jichi Medical University, Saitama, Japan), a graphical user interface for R (The R Foundation for Statistical Computing, Vienna, Austria) (12). The relative tumor volumes and relative mice body weight are presented as mean \pm SD. Tukey-Kramer analysis was used for comparisons among the three groups. Differences with a probability value of $p \leq 0.05$ were considered statistically significant.

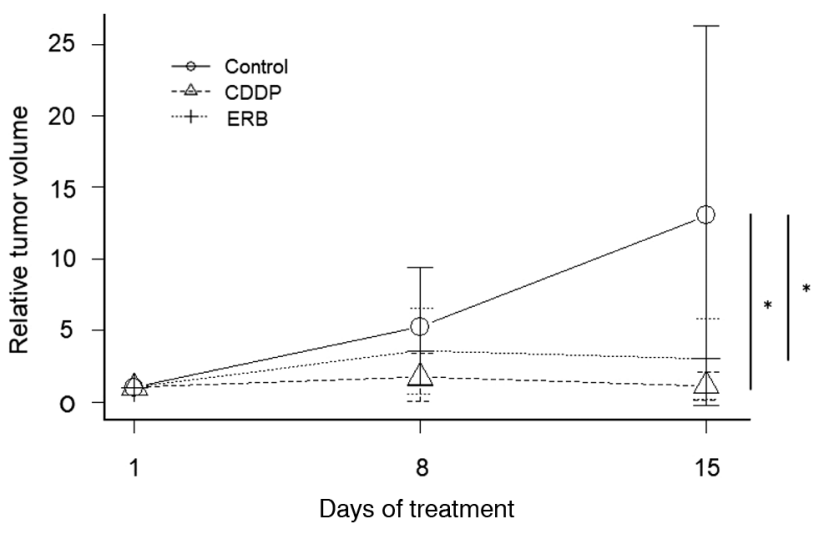

Figure 2. Efficacy of drugs on the breast osteosarcoma-PDOX. Line graphs show tumor volume at the indicated times relative to that at the start of treatment. ${ }^{*} \leq \leq 0.05$. Error bars: $\pm S D$. CDDP: cisplatinum; ERB: eribulin.

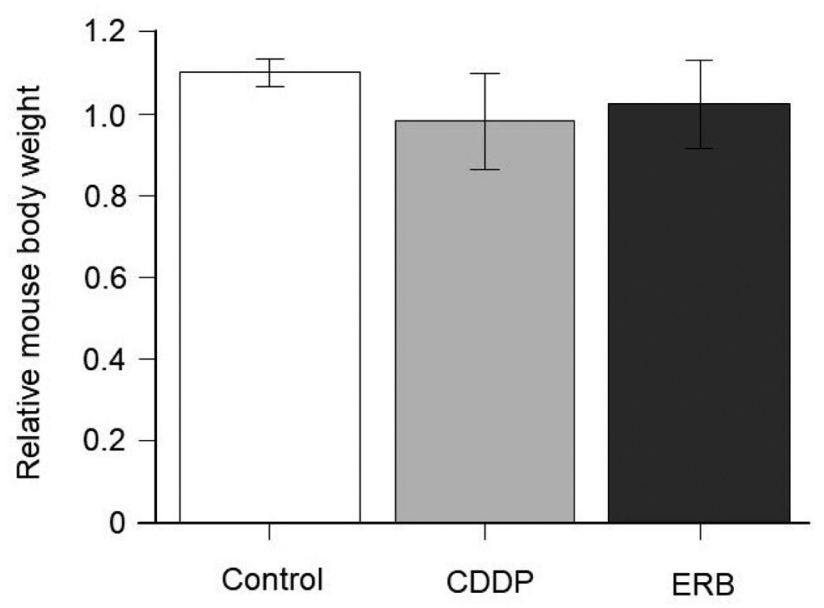

Figure 3. Mouse body weight. Bar graphs show body weight of mice from each group at day 15 relative to day 1 of treatment.

\section{Results}

Treatment efficacy on the breast osteosarcoma-PDOX. There was a significant difference between the control group and the CDDP group, and between the control group and the ERB group ( $p=0.036, p=0.046$, respectively), but there was no significant difference between the CDDP group and the ERB group ( $p=0.964)$ (Figure 2). There was no significant difference in any group in the body weight of mice on the 1 st day and 15 th day (Figure 3).

Histology of breast osteosarcoma-PDOX in H\&E-stained sections obtained from paraffin-embedded formalin-fixed fixed tissues. In the untreated-control group, pleomorphic spindle cells 

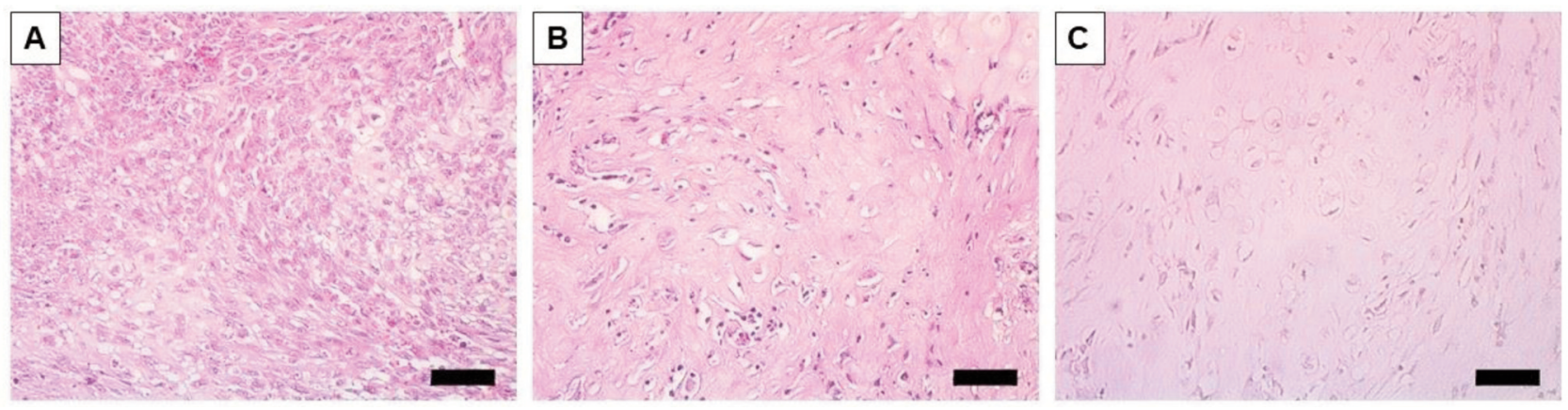

Figure 4. Representative photomicrographs of $H \&$ E-stained breast-osteosarcoma PDOX tissue sections. (A) Untreated control. (B) Cisplatinumtreated. (C) Eribulin-treated. Scale bar: $50 \mu \mathrm{m}$.

were observed, and some of them showed increased nuclear mitosis. In addition, malignant osteoid formation was observed in the stroma in the control mice (Figure 4A). In contrast, the CDDP- and ERB-treatment groups showed an obvious decrease in the density of pleomorphic spindle cells (Figure 4B and C).

\section{Discussion}

Extraskeletal osteosarcoma accounts for about $4 \%$ of all osteosarcomas (13). While osteosarcoma of bone origin occurs mainly in the first two decades of life, extraskeletal osteosarcoma mainly affects the elderly, aged 65 and older (14). The mechanism by which osteosarcoma develops exosteographically is not well understood. Primary osteosarcoma of the mammary gland has been suggested to originate from mesenchymal cells within the mammary gland, or to be the result of excessive development of mesenchymal cancer cells such as fibroadenoma or phyllodes tumor $(1,15)$. Chemotherapy for osteosarcoma of the breast has been tried mainly with first-line treatment for osteosarcoma of bone origin, including methotrexate, doxorubicin (DOX), CDDP, and ifosfamide, either alone or in combination (16). However no first-line therapy has been established for breast osteosarcoma. In the PDOX model, tumors are growing in the mammary gland of mice, making it a clinically-relevant model and a candidate for clinical treatment can possibly be identified. Our recent studies have also shown that ERB is effective for tumor regression in a bone-derived osteosarcoma PDOX model (10) and in CDDP- and DOXresistant lung metastatic osteosarcoma (17). ERB has been reported in recent studies to be combined with a wide range of anticancer agents (18). ERB's anti-tumor mechanism of action is based on the modulation of the tumor microenvironment and vascular remodeling (19).

Although in the present study ERB did not show greater efficacy than CDDP, the present study suggests that both ERB and CDDP have promise as first-line treatment for osteosarcoma of mammary origin.

\section{Conflicts of Interest}

The Authors declare that they have no conflicts of interest in relation to this study. AntiCancer Inc. uses PDOX models for contract research.

\section{Author's Contributions}

N.M. and N.F.W. conceived the study, N.M. and Y.A. performed the experiments and J.Y., J.M. and R.M.H. provided scientific advice. N.M. wrote the paper and R.M.H. revised the paper. R.M.H. supervised the study.

\section{Acknowledgements}

This paper is dedicated to the memory of A. R. Moossa, M.D., Sun Lee, M.D., Professor Li Jiaxi and Masaki Kitajima, M.D.

\section{Funding}

The Robert M. Hoffman Foundation for Cancer Research provided funds for the study.

\section{References}

1 Silver SA and Tavassoli FA: Primary osteogenic sarcoma of the breast: a clinicopathologic analysis of 50 cases. Am J Surg Pathol 22(8): 925-933, 1998. PMID: 9706972. DOI: 10.1097/ 00000478-199808000-00002

2 Pollard SG, Marks PV, Temple LN and Thompson HH: Breast sarcoma. A clinicopathologic review of 25 cases. Cancer 66(5): 941-944, 1990. PMID: 2386920. DOI: 10.1002/10970142(19900901)66:5<941::aid-cncr2820660522>3.0.co;2-b

3 Kennedy T and Biggart JD: Sarcoma of the breast. Br J Cancer 21(4): 635-644, 1967. PMID: 4294611. DOI: 10.1038/bjc.1967.74

4 Allemani C, Matsuda T, Di Carlo V, Harewood R, Matz M, Nikšić M, Bonaventure A, Valkov M, Johnson CJ, Estève J, Ogunbiyi OJ, Azevedo E Silva G, Chen WQ, Eser S, Engholm G, Stiller CA, Monnereau A, Woods RR, Visser O, Lim GH, Aitken J, Weir HK, Coleman MP and CONCORD Working 
Group: Global surveillance of trends in cancer survival 2000-14 (CONCORD-3): analysis of individual records for 37513025 patients diagnosed with one of 18 cancers from 322 populationbased registries in 71 countries. Lancet 391(10125): 1023-1075, 2018. PMID: 29395269. DOI: 10.1016/S0140-6736(17)33326-3

5 Kelland L: The resurgence of platinum-based cancer chemotherapy. Nat Rev Cancer 7(8): 573-584, 2007. PMID: 17625587. DOI: $10.1038 / \mathrm{nrc} 2167$

6 Smrke A, Anderson PM, Gulia A, Gennatas S, Huang PH and Jones RL: Future directions in the treatment of osteosarcoma. Cells 10(1): 172, 2021. PMID: 33467756. DOI: 10.3390/cells 10010172

7 Dumontet $\mathrm{C}$ and Jordan MA: Microtubule-binding agents: a dynamic field of cancer therapeutics. Nat Rev Drug Discov 9(10): 790-803, 2010. PMID: 20885410. DOI: 10.1038/nrd3253

8 Smith JA, Wilson L, Azarenko O, Zhu X, Lewis BM, Littlefield BA and Jordan MA: Eribulin binds at microtubule ends to a single site on tubulin to suppress dynamic instability. Biochemistry 49(6): 1331-1337, 2010. PMID: 20030375. DOI: 10.1021/bi901810u

9 Miwa S, Yamamoto N, Hayashi K, Takeuchi A, Igarashi K and Tsuchiya $\mathrm{H}$ : Therapeutic targets for bone and soft-tissue sarcomas. Int J Mol Sci 20(1): 170, 2019. PMID: 30621224. DOI: $10.3390 /$ ijms 20010170

$10 \mathrm{Wu}$ NF, Yamamoto J, Aoki Y, Bouvet M and Hoffman RM: Eribulin inhibits osteosarcoma in a clinically-accurate bone-tumorinsertion PDOX mouse model. Anticancer Res 41(4): 1779-1784, 2021. PMID: 33813382. DOI: 10.21873/anticanres.14943

11 Wu NF, Wu J, Yamamoto J, Aoki Y, Hozumi C, Bouvet M and Hoffman RM: The first mouse model of primary osteosarcoma of the breast. In Vivo 35(4): 1979-1983, 2021. PMID: 34182472. DOI: 10.21873 /invivo. 12466

12 Kanda Y: Investigation of the freely available easy-to-use software 'EZR' for medical statistics. Bone Marrow Transplant 48(3): 452458, 2013. PMID: 23208313. DOI: 10.1038/bmt.2012.244

13 Allan CJ and Soule EH: Osteogenic sarcoma of the somatic soft tissues. Clinicopathologic study of 26 cases and review of literature. Cancer 27(5): 1121-1133, 1971. PMID: 5281245. DOI: 10.1002/ 1097-0142(197105)27:5<1121::aid-cncr2820270519>3.0.co;2-3
14 Vorobiof G, Hariparsad G, Freinkel W, Said H and Vorobiof DA: Primary osteosarcoma of the breast: a case report. Breast J 9(3): 231-233, 2003. PMID: 12752633. DOI: 10.1046/j.15244741.2003.09320.x

15 Remadi S, Doussis-Anagnostopoulu I and Mac Gee W: Primary osteosarcoma of the breast. Pathol Res Pract 191(5): 471-4; discussion 475-7, 1995. PMID: 7479366. DOI: 10.1016/S03440338(11)80737-5

16 Momoi H, Wada Y, Sarumaru S, Tamaki N, Gomi T, Kanaya S, Katayama $\mathrm{T}$, Ootoshi $\mathrm{M}$ and Fukumoto $\mathrm{M}$ : Primary osteosarcoma of the breast. Breast Cancer 11(4): 396-400, 2004. PMID: 15604996. DOI: 10.1007/BF02968048

17 Kiyuna T, Tome Y, Miyake K, Murakami T, Oshiro H, Igarashi K, Kawaguchi K, Hsu J, Singh M, Li Y, Nelson S, Bouvet M, Singh SR, Kanaya F and Hoffman RM: Eribulin suppressed cisplatinum- and doxorubicin-resistant recurrent lung metastatic osteosarcoma in a patient-derived orthotopic xenograft mouse model. Anticancer Res 39(9): 4775-4779, 2019. PMID: 31519578. DOI: 10.21873/anticanres.13661

18 Asano M, Matsui J, Towle MJ, Wu J, McGonigle S, DE Boisferon MH, Uenaka T, Nomoto K and Littlefield BA: Broadspectrum preclinical antitumor activity of eribulin (Halaven $($ ): combination with anticancer agents of differing mechanisms. Anticancer Res 38(6): 3375-3385, 2018. PMID: 29848686. DOI: 10.21873/anticanres.12604

19 Ito K, Hamamichi S, Abe T, Akagi T, Shirota H, Kawano S, Asano M, Asano O, Yokoi A, Matsui J, Umeda IO and Fujii H: Antitumor effects of eribulin depend on modulation of the tumor microenvironment by vascular remodeling in mouse models. Cancer Sci 108(11): 2273-2280, 2017. PMID: 28869796. DOI: $10.1111 /$ cas. 13392
Received August 24, 2021

Revised September 10, 2021 Accepted September 13, 2021 